\title{
Incidencia y severidad de Mal de Panamá (Fusarium Sp) en Guineo (Musa balbisiana ABB)
}

\author{
Incidence and severity of Panama Disease (Fusarium Sp) on Guineo (Musa balbisiana ABB)
}

Trinidad Castillo- Arévalo ${ }^{1}$

\section{Resumen}

El cultivo de las musáceas (Musaceae) en Nicaragua, ha sido un rubro de buena rentabilidad y aceptables índices de producción, representa un producto promisorio en el departamento de Rivas y en algunas zonas del país. Los ingresos de este cultivo se han visto amenazados por esta enfermedad (Mal de Panamá) que afecta a la planta y sus frutos. Con el objetivo de contribuir al conocimiento científico, a través de un estudio de los principales síntomas y condiciones ambientales de Mal de Panamá, asociados al cultivo de guineo, se realizó esta investigación de marzo del 2014 a marzo del 2015, en un sistema Taungya, para describir los principales síntomas de daño y calcular la incidencia, severidad y ABCPE (Área Bajo la Curva de Progreso de la Enfermedad) de Mal de Panamá en cultivo de guineo.

La recolección de los datos del patógeno se hizo a través de monitoreos visuales en campo y diagnóstico de laboratorio de la Universidad Nacional Agraria. El muestreo se realizó semanal, para la prueba de campo se tomaron muestras de plantas enfermas o sintomatológicas del agente causal, se procedió a su eliminación. Como resultado del estudio se identificó Fusarium sp asociado al cultivo de guineo. De manera general la incidencia de este agente causal reflejó un comportamiento similar en los bloques de guineo (o.53\%) y caoba (0.61\%), seguido de melina (0.36 \%), en los bloques de cedro, pochote, roble y teca los síntomas de marchitamiento vascular se presentaron en porcentajes de $1.12 \%$ a $1.80 \%$.

Palabras clave: Fusariosis, taungya, guineo.

\section{Abstract}

The cultivation of musaceae (Musaceae) in Nicaragua has been an area of good profitability and acceptable production rates, representing a promising product in the department of Rivas and in some areas of the country. The income of this crop has been threatened by this disease that affects the plant and its fruits. With the objective of contributing to scientific knowledge through a study of the main symptoms and environmental conditions of Panama Disease, associated with the guineo crop,

1 MSc en Sanidad Vegetal, Docente-Investigador de la Universidad Nacional Agraria (UNA). Correo: trinidad.castillo@ci.una.edu.ni, ORCID: https://orcid.org/oooo-0001-6401-0142

Recibido: 4/10/2021 - Aprobado: 26/11/2021 
this research was conducted from March 2014 to March 2015, in a Taungya system, to describe the main symptoms of damage and calculate the incidence, severity and $\mathrm{ABCPE}$ of Panama Disease in guineo crop.

Pathogen data collection was done through visual monitoring in the field and laboratory diagnosis at the National Agrarian University. Sampling was carried out weekly; for the field test, samples of diseased or symptomatic plants of the causal agent were taken and eliminated. As a result of the study, Fusarium sp associated with the guineo crop was identified. In general, the incidence of this causal agent showed a similar behavior in the guineo ( $0.53 \%$ ) and mahogany ( $0.61 \%)$ blocks, followed by melina ( $0.36 \%$ ), the cedar, pochote, oak and teak blocks showed vascular wilt symptoms in percentages ranging from $1.12 \%$ to $1.80 \%$.

Keywords: fusarium wilt, taungya, guineo.

\section{Introducción}

Los guineos, bananos y plátanos (Musa spp.) están entre los cultivos más importantes en los países del trópico y el subtrópico. Estos ocupan el cuarto lugar en importancia a escala mundial después del arroz, el trigo y el maíz (FAO, 2017). Sin embargo, la producción de estos cultivos se encuentra amenazada por el ataque de enfermedades como el Mal de Panamá o marchitez por Fusarium. Esta enfermedad causada por Fusarium oxysporum f. sp. cubense (FOC) representa una de las más destructivas y de importancia económica en el género Musa (Lara, 2009 y Ploetz, 2015).

En la actualidad, la enfermedad Fusariosis que afecta especies del género musa, continúa siendo un problema porque puede permanecer en suelos bananeros infectados por más de 30 años, destruyendo cultivares susceptibles a la raza 1 como el cultivar Gros Michel (AAA), establecido por pequeños y medianos productores de Nicaragua y Costa Rica (Caballero et al., 2011).

Inicialmente se observa clorosis en las hojas más viejas, el amarillamiento comienza a lo largo de los márgenes de la hoja, según avanza llega a la vena central, el pecíolo de la hoja colapsa. Los síntomas internos se caracterizan por manchas marrón - rojizo en el sistema vascular de los rizomas, cormos y pseudotallos, según la enfermedad avanza las hojas más jóvenes se afectan y mueren formándose una falda de hojas muertas alrededor del tallo (Alvarado y Díaz, 2007 y Shew y Lucas, 1991).

El patógeno es un organismo que habita en el suelo y que sobrevive entre los cultivos en los restos de plantas infectados que yacen en el suelo en forma de micelio y en cualquiera de sus formas de esporas, pero lo hace con mayor frecuencia en forma de clamidosporas, sobre todo en las regiones templadas frías. Se propaga a cortas distancias a través del agua y el equipo agrícola contaminado, y a grandes distancias 
principalmente en los trasplantes infectados o en el suelo que va en ellos. Es frecuente que una vez que un área haya sido infectada por fusarium se mantenga así por tiempo indefinido (Ochoa et al., 2004).

Ploetz y Correll (1988) aseguran que independientemente de su origen, el agua constituye un medio excelente de transporte de las esporas y por lo tanto una inmejorable vía de infección.

Caballero et al. (2011) indican obtención de inóculo de FOC de raíces estériles de la maleza Commelina diffusa.

\section{Materiales y métodos}

Descripción del estudio desarrollado. El trabajo se realizó en la finca Santa María, ubicada en la comunidad la Chocolata de Rivas, situada entre las coordenadas $11^{\circ} 41^{\prime}$ Norte, $85^{\circ} 83^{\prime}$ longitud Oeste, a $120 \mathrm{~km}$ de Managua, carretera panamericana Sur y a $54 \mathrm{~km}$ de Peñas Blancas (frontera con Costa Rica).

Área de estudio. El trabajo de investigación se estableció en un sistema Taungya, en un área de 1,o ha, en el que se desarrolla la investigación sobre "Evaluación del crecimiento, potencial de secuestro y fijación de carbono de seis especies forestales en asocio con Mussa balbisiana ABB".

Las especies forestales se establecieron en julio 2012 con distancia de siembra de 6 x 1,5 m (110o plantas ha) y el guineo se estableció en julio 2013, entre las calles de las especies forestales con el mismo marco de siembra (1100 plantas ha).

Las especies forestales establecidas en el estudio fueron, Teca (Tectona grandis), Pochote (Bombacopsis quinata), Roble (Tabebuia rosea), Melina (Gmelina arbórea), Cedro real (Cedrela odorata) y Caoba (Swietenia humilis) cada una de ellas se establecieron con y sin asocio de Guineo (Musa balbisiana $A B B$ ).

Ubicación de los tratamientos. El área está conformada por tres bloques y 16 parcelas experimentales, en el área de las parcelas asociadas la cantidad de plantas de guineo y especies forestales es de 40 y en las parcelas sin asocio de 24 plantas. Para este estudio solo se trabajó con los bloques 1,2 y 3.

La distancia de siembra de guineo en el área del asocio con las especies forestales correspondió a $1.5 \times 6 \mathrm{~m}$ para una densidad poblacional por parcela experimental de 40 plantas y 1,111 plantas ha y en el área sin asocio el marco de siembra fue de $3 \times 3 \mathrm{~m}$ para una densidad poblacional de 24 plantas por parcela experimental y 1,111 plantas ha. 
Diseño experimental. El trabajo de investigación se estableció bajo el diseño de bloque incompleto balanceado (González, 2006).

Descripción del estudio. La recolección de los datos sobre la incidencia de Fusarium oxysporum se hizo a través de monitoreos visuales en campo (sintomáticos) y análisis de laboratorio.

Monitoreos. El monitoreo consistió en seleccionar todos los surcos de los tres bloques con guineo, en los cuales se recorrían y se observaban las 576 plantas. Como estaba codificado el número de plantas e hileras, las plantas con síntomas iniciales se marcaban con una cinta plástica de color para darle seguimiento a los síntomas progresivos de las enfermedades en los monitoreos posteriores, verificando de este modo el avance de estos.

Estos monitoreos fueron acompañados con tomas de fotografías, lo que documenta la información pertinente de la enfermedad.

Frecuencia de los monitoreos. Se realizaron cada 7 días, iniciando en la segunda quincena de marzo del 2014 y finalizando en la segunda quincena de marzo del 2015.

Muestreo de plantas enfermas. Para la prueba de campo se tomaron muestras de plantas enfermas o que presentaban las características sintomatológicas del agente causal estudiado, esta se levantaba en una base de datos que llevaba, para esto se cortaba una o dos hojas y se observaba la vascularización de la nervadura, si presentaba síntomas de estrías o rayas rosadas-amarillas o se tornaban café y finalmente negras, al mismo tiempo se procedió a su eliminación, se trasladaban las plantas a un área fuera de la plantación y se cortaron porciones de pseudotallo, vainas, hojas, pedúnculo, y frutos de aproximadamente 5 a $15 \mathrm{~cm}$ de longitud, de esa misma muestra se depositaban dentro de un termo con hielo para el transporte del campo a los laboratorios de fitopatología de la Universidad Nacional Agraria, Managua.

Evaluación de la incidencia. La incidencia de la enfermedad se calculó por el número de plantas que presentaron síntomas y se expresó en porcentajes de plantas enfermas según Caballero et al. (2011)

$$
\text { Incidencia (\%) }=\frac{\text { número de hojas muestreadas con sintomas }}{\text { Total de hojas muestreados }} \times 100
$$

Severidad. Con los valores de la severidad se calculó el índice de la enfermedad descrito por McKinney y Davis (1923), utilizando la siguiente fórmula:

$$
\mathrm{XXE}=\frac{\sum \text { (número de plantas enfermas } \mathrm{x} \text { cada grado de la escala) }}{\text { (Número total de plantas observadas) } \times(\text { grado mayor de la escala) }} \quad \times \quad \text { 100 }
$$


Cuadro 1: Escala de severidad de Fusarium (Mal de Panamá) descrito por McKinney y Davis (1923)

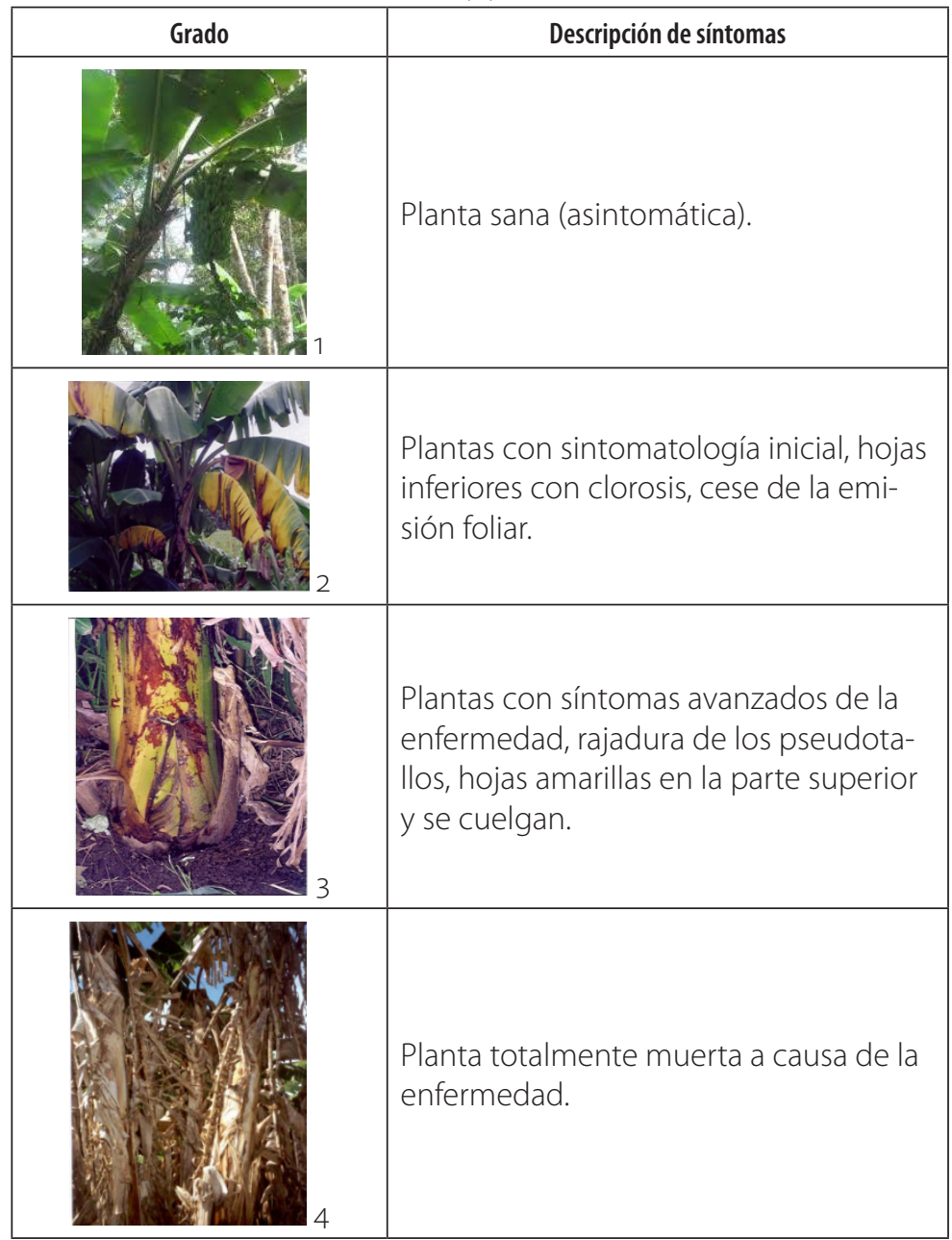

Modificado por: Trinidad Castillo- Arévalo.

\section{Cálculo del Área Bajo la Curva de Progreso de la Enfermedad (ABCPE) para Fusariosis}

Con los registros de severidad por tratamiento se calculó el Área Bajo la Curva de Progreso de la Enfermedad (ABCPE). La fórmula utilizada fue la propuesta por (Shaner y Finney, 1977).

$$
\mathrm{ABCPE}=\sum_{i=1}^{n-1}\left[\frac{X_{i+1}+X_{i}}{2}\right]\left(t_{i+1}-t_{i}\right)
$$


$x_{i}=$ Porcentaje de tejido afectado

$t=$ Tiempo (días)

$n=$ número de evaluaciones

\section{Identificación y descripción de fusarium en laboratorio}

Análisis patológico de material vegetativo. Previo a la identificación y descripción se realizó el análisis patológico del material vegetativo, se utilizaron técnicas de inducción de crecimiento de hongos, a partir de tejido vegetal enfermo posteriormente se sembró en medios de cultivos como: Agar-Agua (AA) y Papa Dextrosa Agar (PDA) para inducir la esporulación de estructuras reproductivas del patógeno.

Cámara húmeda. Las muestras de tejido vegetal enfermo (hojas) se colocaron en platos petri o cajas plásticas con papel filtro, humedecido con agua destilada estéril para inducir a la esporulación de estructuras fructíferas de los hongos y su posterior identificación mediante el uso de microscopio.

Siembra de trozos de hojas con estructuras fructíferas y tejido infectado en AA y PDA. Se tomaron muestras de tejido con síntomas, se realizaron pequeños cortes en el tallo de manera que solo quedara en la lesión la estructura, luego se dejó reposar en agua destilada estéril por un minuto, se secó con papel filtro y se dejó reposar por 30 segundos, finalmente se sembró en platos Petri. Este procedimiento es el de inducir el desarrollo y crecimiento de estructuras de reproducción. Para la siembra de trozos de tallo con tejido infectado en medio de cultivo Papa Dextrosa Agar (PDA), las muestras fueron primeramente desinfectadas con hipoclorito de sodio al $1 \%$ y alcohol histólico por 2 minutos, luego se enjuagó con ADE (Agua Destilada Estéril), se hicieron un total de 4 réplicas por un lapso de tiempo de 15 días, posteriormente se sembraron en platos Petri con PDA; estos platos petri se rotularon de la siguiente manera: nombre del cultivo, fecha de muestreo y nombre de la parcela.

Finalmente, los platos se preservaron a temperaturas de 25-30 grados centígrados. Estos platos se revisaban semanalmente con el propósito de observar estructuras reproductivas.

Identificación de Fusarium Sp. El género de hongo encontrado fue identificado utilizando claves taxonómicas. Las características morfológicas o de crecimiento se observaron en microscopio (Monterrosa, 1996).

Modelo lineal. Caballero (1985). El modelo lineal para un bloque incompleto balanceado con estructura de bloque al azar es:

$$
Y_{i j}=\mu+\tau_{i}+\rho_{j}+e_{i j}, i=1,2, \ldots, t_{j}=1,2, \ldots, b
$$




$$
\begin{aligned}
& \mu=\text { Media general } \\
& \tau_{i}=\text { Efecto del i-esimo tratamiento } \\
& p_{j}=\text { Efecto del j-esimo bloque } \\
& e_{i j}=\text { Error experimental } \\
& \sigma^{2}=\text { Varianza }
\end{aligned}
$$

\section{Análisis de los datos}

Después de haber colectado los datos en el campo, se procedió a ordenarlos por variables en una tabla de datos en Excel, luego cada variable medida fue comparada entre sitios usando análisis (ANDEVA) (InfoStat, 2008) seguido de un análisis de diferencia mínima significativa (LSD). El nivel de significancia a usar fue de ( $p \leq 0.05)$. Para el análisis de varianza se realizaron transformaciones de datos para ajustar los supuestos de ANDEVA. Se utilizó logaritmo en base $10 \log (\mathrm{x})$. Para incidencia y severidad en InfoStat (2008).

\section{Variables:}

- Porcentaje de Incidencia de daño de Fusarium $S p$

\begin{tabular}{|c|c|}
\hline \multicolumn{2}{|c|}{$\%$ severidad } \\
\hline Fincas & Media \pm ES \\
\hline Melina & $0.36 \pm 0.14 a$ \\
\hline Guineo & $0.53 \pm 0.13 \mathrm{ab}$ \\
\hline Caoba & $0.61 \pm 0.16 \mathrm{ab}$ \\
\hline Cedro & $1.12 \pm 0.25 b c$ \\
\hline Pochote & $1.12 \pm 0.26 b c$ \\
\hline Roble & $1.59 \pm 0.36 c$ \\
\hline Teca & $1.80 \pm 0.42 \mathrm{C}$ \\
\hline C.V & 16.36 \\
\hline $\mathrm{p}$ & 0.0001 DS \\
\hline$F ; d f ; n$ & $2.96 ; 130 ; 2983$ \\
\hline
\end{tabular}

- Índice de severidad de Fusarium Sp

- $\mathrm{ABCP}$ Fusarium $S p$

\section{Resultados y discusión}

Cuadro 2: Análisis de varianza del porcentaje de incidencia de Fusariosis

ES $=$ Error estándar, DS = Diferencia Significativa, C.V = Coeficiente de Variación, $\mathrm{P}=$ Probabilidad según Duncan, $\mathrm{F}=$ Fisher calculado df = Grados de libertad del error, $\mathrm{n}=$ Números de datos utilizados en el análisis. ${ }^{*}$ Medias con letras distintas existe diferencias significativas. Fuente: elaboración propia. 


\section{Comparación del porcentaje de incidencia de Fusariosis (Fusarium sp.)}

En el análisis de la varianza, se observó diferencia significativa (= $\leq 0.05)$. De manera general la incidencia de este agente causal reflejó un comportamiento similar en los bloques de guineo $(53 \%)$ y caoba $(61 \%)$, seguido de melina $(36 \%)$, los bloques de cedro, pochote, roble y teca los síntomas de marchitamiento vascular se presentaron porcentajes de $1.12 \%$ a $1.80 \%$.

De igual manera Pérez et al. (2013) en su investigación: Efecto del Bio-carbón sobre Fusarium oxysporum f. sp. Cubense y el desarrollo de plantas de banano (Musa $\mathrm{AAA}$ ), el patógeno presentó síntomas característicos, además en estudios realizados por Scott et al. (2012), En marchitamiento vascular en lechuga, ocasionado por F. o. f. sp. Lactucae, se observó que este agente causal reflejó un comportamiento similar al que se mostró en este estudio.

\section{Comparación del porcentaje de incidencia de Mal de Panamá (Fusarium sp.) en el cultivo de guineo}

De manera general se observa que la incidencia de este agente causal se presentó casi en todos los meses de muestreo, sin embargo, el mayor aumento de la enfermedad ocurrió treinta días después del mayor aumento de precipitaciones, esto creó las condiciones favorables para la reproducción del patógeno lo cual permitió registrar la mayor afectación en los meses de noviembre y diciembre del 2014 a $0.53 \%$ de incidencia. Ochoa et al. (2004), en su estudio Dos nuevas formas de especie Fusarium oxysporum, que causa marchitez vascular, y Robles-Carrión et al. (2016) en su estudio Identificación morfológica y molecular de especies de Fusarium asociadas a la marchitez vascular del tabaco (Vasconcellea heilbornii var. pentagona Badillo) demuestran que los síntomas de Fusarium oxysporum aparecen al mes de la infección, o sea que se relaciona con el comportamiento del patógeno en este estudio.

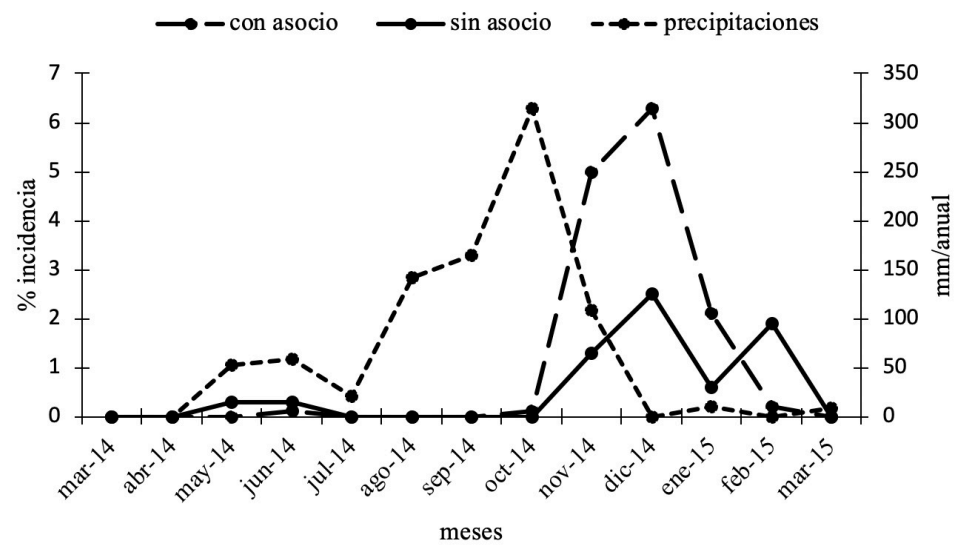

Figura 1: Porcentaje de incidencia de Fusariosis. Fuente: elaboración propia. 
Cuadro 3: Análisis de varianza del porcentaje de incidencia de Fusariosis

\begin{tabular}{|l|l|}
\hline \multicolumn{2}{|c|}{ \% severidad } \\
\hline \multicolumn{1}{|c|}{ Fincas } & \multicolumn{1}{c|}{ Media \pm ES } \\
\hline Melina & $0.41 \pm 0.13 \mathrm{a}$ \\
\hline Guineo & $0.13 \pm 0.03 \mathrm{a}$ \\
\hline Caoba & $0.25 \pm 0.09 \mathrm{ab}$ \\
\hline Cedro & $0.43 \pm 0.59 \mathrm{bc}$ \\
\hline Pochote & $0.38 \pm 0.08 \mathrm{bc}$ \\
\hline $\begin{array}{l}\text { Roble } \\
\text { Teca }\end{array}$ & $\begin{array}{l}0.53 \pm 0.10 \mathrm{bc} \\
0.92 \pm 0.18 \mathrm{C}\end{array}$ \\
\hline C.V & 12.33 \\
\hline $\mathrm{P}$ & $0.0001 \mathrm{DS}$ \\
\hline F; df; $\mathrm{n}$ & $44.58 ; 134 ; 2983$ \\
\hline
\end{tabular}

ES $=$ Error estándar, DS = Diferencia Significativa, $C . V=$ Coeficiente de Variación, $\mathrm{P}=$ Probabilidad según Duncan, $\mathrm{F}=$ Fisher calculado df = Grados de libertad del error, $\mathrm{n}=$ Números de datos utilizados en el análisis. * Medias con letras distintas existe diferencias significativas. Fuente: elaboración propia.

\section{Comparación del porcentaje de severidad de Fusariosis (Fusarium sp.)}

En el análisis de la varianza, se observó diferencia significativa $(=\leq 0.05)$. De manera general la severidad de este agente causal reflejó un comportamiento similar en los bloques de melina (o.41\%), cedro (o.43\%), seguido de pochote (o.38\%), debido a que el asocio con estas especies forestales generan mayor cantidad de sombra y por lo tanto se desarrolla en mayor porcentaje la epifítia, en el estudio realizado por Barrera et al. (2016): Efecto del sombrío sobre la sigatoka negra (Mycosphaerella fijiensis Morelet) en cultivo de plátano cv hartón (Musa AAB Simmonds) en el Caribe húmedo de Colombia, demuestran que el efecto de asocio de forestales con musáceas desarrolla el porcentaje de severidad de los patógenos de origen fungosos, como lo es Fusariosis, coincidiendo el comportamiento del patógeno en nuestro estudio.

Al mismo tiempo Martínez (2003) en su investigación El cultivo de plátano en los llanos orientales en Colombia, demuestra que en las musáceas bajo condiciones de sombrío, el patógeno se desarrolla en altos porcentajes como sucedió en esta investigación. 


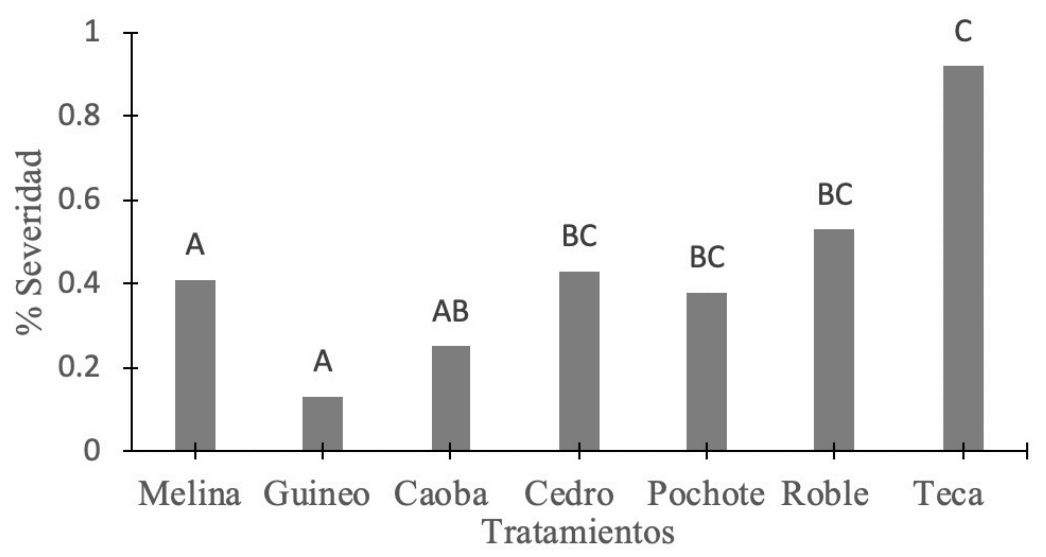

Figura 2: Porcentaje de severidad de Fusariosis. Fuente: elaboración propia

\section{Comparación del porcentaje de severidad de Fusariosis (Fusarium sp.)}

En el análisis de la varianza, se observó diferencia significativa (= $\leq 0.0001)$. Los tratamientos guineo (o.13 \%) y teca (0.92\%) son los que presentan menor y mayor porcentaje de severidad en la investigación, el tratamiento guineo, va solo, no hay efecto sombrío razón por la cual el porcentaje de afectación del patógeno es menor y el asocio de guineo con teca la epifítia asciende hasta $0.92 \%$ debido a la cantidad de sombra que genera esta planta forestal en asocio con guineo, investigaciones realizadas por Martínez (2003) en su estudio El cultivo del plátano en los llanos orientales y Almodóvar y Díaz (2007) en su investigación de identificación y manejo de sigatoka negra y otras enfermedades de plátano y guineo, señalan que los diferentes niveles de sombrío obtenidos en cultivos de forestales asociados con plátano, libre exposición solar, fueron más sensibles a la sigatoka negra, en los resultados de ambas investigaciones coinciden con el comportamiento de Fusariosis en esta investigación.

\section{Comparación del área bajo la curva del progreso de la enfermedad (ABCPE) de Fusariosis}

Se comparó el área bajo la curva de progreso de Fusariosis en guineo, se presenta en la Figura 3. La mayor área bajo la curva se presentó en el bloque del asocio de guineo con teca, seguido de roble y cedro, esto significa mayor oportunidad o mejores condiciones de la enfermedad para que progrese una epifítia mucho más rápido en estos tratamientos con mayor efecto sombrío, en cambio la menor área bajo la curva se presentó en el asocio de guineo con caoba. 


\section{AGROPECUARIA}

Berruezo (2018) en los resultados de su investigación "Caracterización morfológica, biológica y molecular de los complejos Fusarium oxysporum y Fusarium solani asociados al cultivo de Nicotiana tabacum L. en el Noroeste Argentino", coincide que el comportamiento del ABCPE asciende en condiciones de humedad relativa, al igual que esta investigación.

Además, Castillo-Arévalo y Jiménez-Martínez (2020), en su investigación: Incidencia y severidad de enfermedades asociadas al cultivo de plátano (Musa paradisiaca L.) en Rivas, Nicaragua, describen el aumento del comportamiento del patógeno mediante $\mathrm{ABCPE}$ en fincas de mayores condiciones de humedad, lo que favorece el progreso una epifítia mucho más rápido en fincas con mayor humedad relativa.

Al realizar el análisis de varianza y separación de medias para comparar los promedios de ABCPE de Fusariosis, se encontró diferencias significativas $(=\leq 0.05)$.

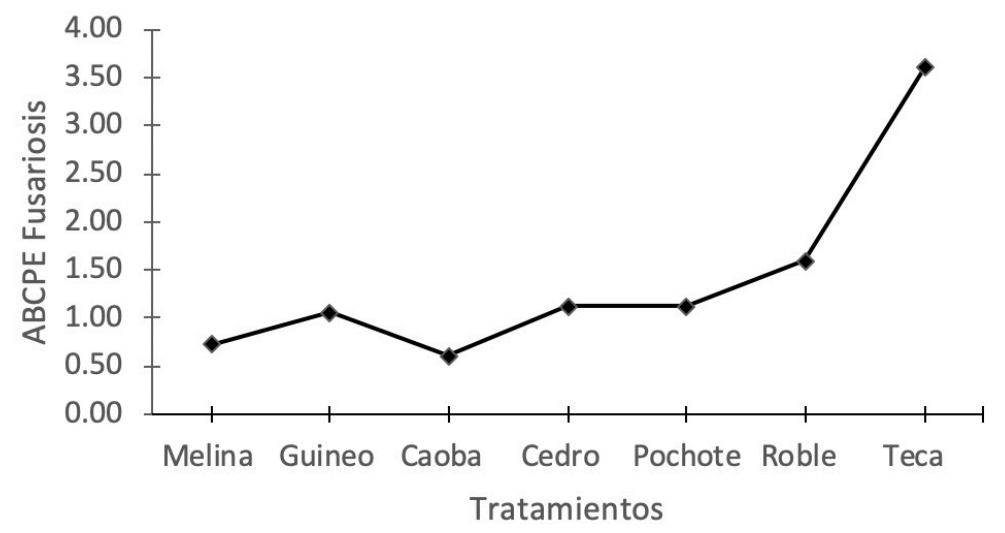

Figura 3: Área bajo la curva de progreso de la enfermedad (ABCPE) de de Fusariosis. Fuente: elaboración propia.

\section{Conclusiones}

Se describió los principales síntomas de daño y se calculó la incidencia, severidad y ABCPE de Mal de Panamá en cultivo de guineo. Se identificó Fusariosis como el principal agente patógeno de enfermedades asociadas al cultivo del guineo en Rivas, Nicaragua.

Se encontró que el mayor pico de crecimiento de la enfermedad ocurrió treinta días después del mayor aumento de precipitaciones, esto creó las condiciones favorables para la reproducción del patógeno lo cual demuestra que los síntomas de Fusarium oxysporum aparecen al mes de la infección. 
Con los resultados de este estudio se demuestra que el efecto de asocio de forestales de mayor producción de sombra con musáceas favorece el desarrollo sobre el incremento del porcentaje de severidad de los patógenos de origen fungosos, como lo es Fusariosis.

Los diferentes niveles de sombrío obtenidos en cultivos de forestales asociados con musáceas, libre exposición solar, son más propensos al desarrollo de Fusariosis, aumentando el $\mathrm{ABCPE}$, lo que favorece el progreso de la epifítia mucho más rápido en fincas con mayor humedad relativa.

\section{Agradecimiento}

El autor de esta investigación agradece a la Universidad Internacional Antonio de Valdivieso (UNIAV), por la financiación económica de este estudio, y a la Universidad Nacional Agraria (UNA) por realizar análisis de laboratorio para identificación del patógeno.

\section{Lista de referencias}

Almodóvar, W., y Díaz, M. (2007). Identificación y manejo de sigatoka negra y otras enfermedades de plátano y guineo. Universidad de Puerto Rico. https://academic. uprm.edu/walmodovar/HTMLobj-276/Manual_ID_y_MIP_de_SN_y_Enferm_ Plat_y_Guineo.pdf

Alvarado, A., y Díaz, M. (2007). Guía práctica de plagas y enfermedades en plátano y guineo. Universidad de Puerto Rico.

Barrera V., J., Barraza A., F., y Campo, A. R. (2016). Efecto del sombrío sobre la sigatoka negra (Mycosphaerella fijiensis Morelet) en cultivo de plátano cv Hartón (Musa AAB Simmonds). Rev. U.D.C.A Act. \& Div. Cient. 19(2), 317-323. https://revistas. udca.edu.co/index.php/ruadc/article/view/85/55

Berruezo, L. A. (2018). Caracterización morfológica, biológica y molecular de los complejos Fusarium oxysporum y Fusarium solani asociados al cultivo de Nicotiana tabacum L. en el Noroeste Argentino [Tesis doctoral, Universidad Nacional de Salta]. Repositorio Institucional CONICET Digital. https://ri.conicet.gov.ar/ handle/11336/80238

Caballero, A., Pocasangre, L., Casanoves, F., Jacques, A.,Tapia A., y Ortiz, J. (2011). Uso de aislamientos endofíticos de Trichoderma spp., para el biocontrol del Mal de Panamá (Fusarium oxysporum f. sp. cubense) raza 1 en vitroplantas de banano del cultivar Gros Michel (AAA) en condiciones de invernadero. La Calera, 13(20), 16-23. https://doi.org/10.5377/calera.v13i20.1620 


\section{AGROPECUARIA}

Caballero, W. (1985). Introducción a la estadística. Editorial IICA.

Castillo-Arévalo, T., y Jiménez-Martínez, E. (2020). Incidencia y severidad de enfermedades asociadas al cultivo de plátano (Musa paradisiaca L.) en Rivas, Nicaragua. La Calera, 20(35). https://doi.org/10.5377/calera.v2oi35.10319

González, I. (2006). Diseños experimentales de bloques incompletos y aplicaciones en la industria [Tesis de pregrado, Universidad Autónoma del Estado de Hidalgo]. UAEH Biblioteca Digital. http://dgsa.uaeh.edu.mx:808o/bibliotecadigital/ bitstream/handle/231104/1766/Dise\%C3\%B10s\%2oexperimentales\%20de\%20 bloques\%2oincompletos\%20y\%20aplicaciones\%20en\%2ola\%2oindustria. pdf? sequence $=1 \&$ isAllowed $=y$

InfoStat. (2008). Statistical Analysis System. (versión 2008) [software].

Lara, D. (2009). Uso de bacterias endofíticas para el control biológico del Mal de Panamá (Fusarium oxysporum f. sp. cubense) en el cultivar Gros Michel (AAA) [Tesis de maestria, Centro agronómico Tropical de Investigación y Enseñanza]. Repositio CATIE. http://hdl.handle.net/11554/4238

Martínez, A. (2003). El cultivo del plátano en los Llanos orientales. Editorial Siglo XX. http:// bibliotecadigital.agronet.gov.co/bitstream/11348/4031/1/20061127152826_ El\%2ocultivo\%2odel\%2oplatano\%2ollanos.pdf

McKinney, H. H., y Davis, R. J. (1923). Influence of soil temperature and moisture on infection of wheat seedlings of Heminthosporium sativum. J. Agric. Res, 31(9), 827 - 840. https://naldc.nal.usda.gov/download/IND43967086/PDF

Monterrosa, D. (1996). Técnicas fitopatológicas de laboratorio para el diagnóstico de las enfermedades de las plantas. Proyecto CATIE INTA-MIP (NORAD).

Ochoa, J., Yangari, B., Ellis, M., y Williams R. (2004). Two new formae specials of Fusarium oxysporum, causing vascular wilt. Biologia Molecular, (39), 10-17.

Organización de las Naciones Unidas para la Alimentación y la Agricultura. (2017). Global programme on banana Fusarium wilt disease: Protecting banana production from the disease with focus on tropical race 4 (TR4). http://www.fao.org/3/ai7921e.pdf

Pérez, R., Salas, A., Tapia, C., Fernández., Soto, G., y Benjamin, T. (2013). Efecto del Bio-carbón sobre Fusarium oxysporum $\mathrm{f}$. sp. cubense y el desarrollo de plantas de banano (Musa AAA). Intersedes, 14 (27). https://www.scielo.sa.cr/pdf/is/ v14n27/ao4v14n27.pdf 
Ploetz, R. C. (2015). Fusarium wilt of banana. Phytopathology, 105, 1512-1521. https:// doi.org/10.1094/PHYTO-04-15-0101-RVW

Ploetz, R. C., y Correll, J. C. (1988). Vegetative Compatibility Among Races of Fusarium oxysporum f. sp. cubense. Plant Disease, 72, 325-328. https://doi.org/10.1094/ PD-72-0325

Robles-Carrión, A., Leiva-Mora, M., Herrera-Isla, L., Sánchez-Rodríguez, A., y TorresGutiérrez, R. (2016). Morphological and molecular identification of Fusarium species associated with vascular wilt of babaco (Vasconcellea heilbornii var. pentagona Badillo). Revista de Protección Vegetal, 31(3), 184-193. http://revistas. censa.edu.cu/index.php/RPV/article/view/849/770

Scott, J. C., Gordon, T., Kirkpatrick, S. C., Koike, S. T., Matheron, M. E, Ochoa, O. E., Truco, M. J. y Michelmore, R.W. (2012). Crop rotation and genetic resistance reduce risk of damage from Fusarium wilt in lettuce. California Agriculture, 66, 20-24. https://doi.org/10.3733/ca.vo66no1p2o

Shaner, G., y Finney, R. (1977). The effect of Nitrogen fertilization on the expression of slow-mildewing resistance in knox wheat. Phytopathology, (67), 1051-1056. https://www.doi.org/10.1094/Phyto-67-1051

Shew, H. D. y Lucas, G. B. (1991). Compendium of tabacco diseases. APS. 